\title{
Towards an analytical formula for the eigenvalues of the Aharonov-Bohm annular billiard
}

\author{
A. J. Fendrik and M. J. Sánchez ${ }^{\mathrm{a})}$ \\ Departamento de Física J. J. Giambiagi, Facultad de Ciencias Exactas y Naturales, \\ Universidad de Buenos Aires. Ciudad Universitaria, 1428 Buenos Aires, Argentina
}

(Received 25 February 2000; accepted for publication 10 November 2000)

We derive an asymptotic formula for the eigenvalues of the Aharonov-Bohm annular billiard (ABAB) that improves and corrects previous estimates. Employing semiclassical arguments we relate the limitations of the procedure to the topology of the classical phase space of the system. (C) 2001 American Institute of Physics. [DOI: 10.1063/1.1341235]

\section{INTRODUCTION}

In recent years, the interest in quantum billiards has increased considerably. Owing to advances in nanotechnology, it has been possible to fabricate small devices in which the carriers are mainly scattered by the boundaries of the sample. ${ }^{1}$ The high resemblance between these systems, commonly referred to as quantum dots, and the quantum billiards, it is very appealing to study the transport properties of ballistic samples employing quantum billiards as models.

Quantum billiards threaded by a magnetic flux $\phi$ are suitable configurations to model problems related to persistent currents. ${ }^{2,3}$ These equilibrium currents are a consequence of the nature of the eigenfunctions' flux sensitivity, which is strictly of the Aharonov-Bohm type. ${ }^{4,5}$ The current, at zero temperature, carried by the level $E_{n}$ is $I \propto \partial E_{n} / \partial \phi$. The first theoretical works on persistent currents have been in one-dimensional (1D) ring geometries, ${ }^{6}$ in two-dimensional (2D) the computations have been performed employing discrete models or cylindrical geometries. ${ }^{7,8}$ In the last case, the mathematical description of the problem is essentially the same as for the ring geometries because the eigenenergies are pure quadratic forms of the two quantum numbers with a functional dependence on the flux that is identical to 1D systems.

To describe the real "rings" employed in the experiments on persistent currents, ${ }^{9}$ it seems to be more suitable to consider the 2D Aharonov-Bohm annular billiard (ABAB) depicted in Fig. 1. The eigenenergies for this system can be numerically determined from the zeros of the cross products of Bessel functions, but a closed analytical formula for the eigenvalues does not exist.

In a recent paper Samandra and Healy introduced an asymptotic formula for the eigenvalues of a charged particle confined in an annular shell in which there was a cylindrically symmetric static magnetic field inside the inner cylinder. ${ }^{10}$ This configuration is equivalent to thread the annular shell by an static magnetic flux $\phi$ and, therefore, equivalent to the ABAB. In this article we present an asymptotic analytical formula for the eigenenergies of the $A B A B$ that corrects and improves the previous one. ${ }^{10}$ Moreover, we will show that the eigenenergies obtained in Ref. 10 fail to describe quantum states that are present at all the energy scales, even in the semiclassical limit. These states are associated with classical orbits that do not hit the inner radius of the ABAB and cannot be described by the asymptotic expansion presented in Ref. 10. We find that the eigenenergies for these states can be obtained, under certain conditions, through the Debye's expansion for the zeros of the Bessel functions of first kind.

The paper is organized as follows. In Sec. II we introduce the system and summarize the results concerning the solutions of the Schrödinger equation. In Sec. III we obtain the asymptotic expansion for the eigenenergies and compare it to the one obtained in Ref. 10. Part of this section

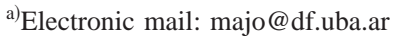




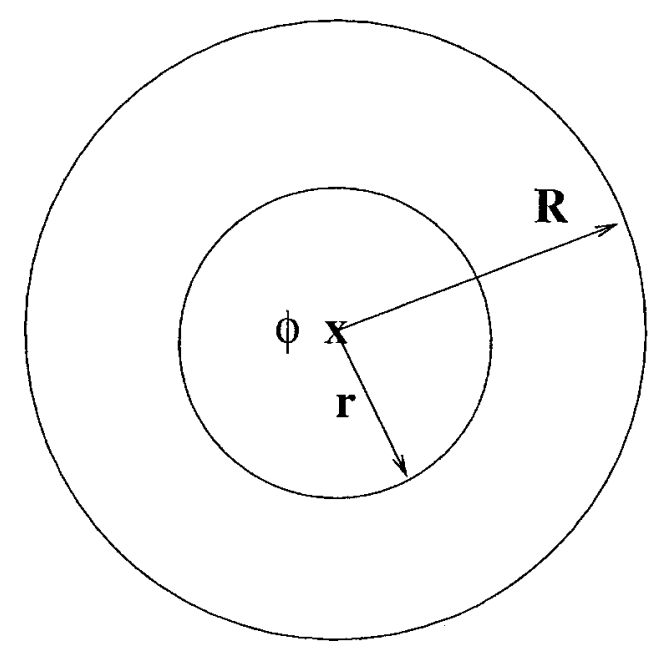

FIG. 1. Aharonov-Bohm annular billiard (ABAB) threaded by a magnetic flux $\phi$. The inner and outer radii are denoted $r$ and $R$, respectively.

is devoted to a detailed discussion about the limitations of the analytical results. We present in Sec. IV the numerical results and in Sec. V the concluding remarks.

\section{THE AHARONOV-BOHM ANNULAR CAVITY}

In this section we introduce the $\mathrm{ABAB}$ and the relevant equations involved in the problem. The annular shell is defined in terms of the polar coordinates $(\rho, \theta)$. The radial coordinate $\rho$ varies between $r$ and $R$ and the azimutal angle $0 \leqslant \theta \leqslant 2 \pi$ (see Fig. 1). We take the area equal to $\pi$ and we define the parameter $\lambda=R / r$, such that $R=\lambda / \sqrt{\lambda^{2}-1}$ and $r=1 / \sqrt{\lambda^{2}-1}$. When $\lambda \rightarrow 1$ the system resembles a $1 \mathrm{D}$ ring, while for $\lambda>1$ it is an annular cavity.

We fix the gauge $\mathbf{A}=\phi /(2 \pi \rho) \hat{\theta}$, where $\hat{\theta}$ is the azimutal unit vector, and there is no magnetic field piercing the body of the annulus.

The single particle spectrum results from the eigenvalue equation

$$
\Delta \Psi+\frac{2 i \alpha}{\rho^{2}} \frac{\partial \Psi}{\partial \theta}-\frac{\alpha^{2}}{\rho^{2}} \Psi+k^{2} \Psi=0
$$

where $\Delta$ is the Laplacian in polar coordinates. We define the scaled flux $\alpha=\phi / \phi_{0}$ with $\phi_{0}$ $=h c / e$ the flux quantum. We use units such $\hbar^{2} / 2 m=1$, so the energy is $E=k^{2}$.

We apply Dirichlet boundary conditions at $\rho=r$ and $\rho=R$ and periodic boundary condition in the azimutal direction. The Eq. (2.1) is separable in polar coordinates and we factorize $\Psi(\rho, \theta)$ $=\mathcal{F}(\rho) \exp (\operatorname{im} \theta)$ with $m=0, \pm 1, \pm 2, \ldots$ the orbital quantum number. The wave numbers $k_{\nu, n}$ result from the solution of the equation

$$
J_{\nu}(z \lambda) N_{\nu}(z)-J_{\nu}(z) N_{\nu}(z \lambda)=0, \quad \nu \equiv m-\alpha,
$$

where we have defined $z \equiv k r$ and $n=1,2 \ldots$, is the radial quantum number. $J_{\nu}$ and $N_{\nu}$ are the Bessel functions of the first and second kind, respectively. The corresponding eigenfunctions $\Psi(\rho, \theta)$ are

$$
\Psi_{\nu, n}(\rho, \theta)=A_{\nu, n} \exp (\operatorname{im} \theta)\left[J_{\nu}\left(k_{\nu, n} \rho\right) N_{\nu}\left(k_{\nu, n} r\right)-J_{\nu}\left(k_{\nu, n} r\right) N_{\nu}\left(k_{\nu, n} \rho\right)\right],
$$

where $A_{\nu, n}$ is the normalization constant. 
All the eigenstates and all the equilibrium physical properties of the system are periodic functions of the flux with period $\phi_{0}$. Moreover, as the energy spectrum is symmetric with respect to $\phi=\phi_{0} / 2$, in the following the parameter $\alpha$ will take values between 0 and $1 / 2$. For $\alpha \neq 0$, the states with $m$ and $-m$ are, in general, not degenerate.

We remark that the eigenenergies $E_{\nu, n}$ cannot be written down as simple functions of the numbers $\nu$ and $n$ as it happens, for example, in the case of the cylindrical geometries where the eigenenergies are pure quadratic functions of both quantum numbers. Moreover, there is not a closed analytical expression for the eigenenergies of the Aharonov-Bohm annular cavity.

\section{THE ASYMPTOTIC EXPANSION FOR THE EIGENENERGIES}

To derive an estimate for the eigenenergies $E_{\nu, n}$, we begin rewriting the asymptotic expansion of the $n$th zero of Eq. (2.2) that appears in Ref. 11, in such a way that the dependence on $n$, that is hidden in the expression given in Ref. 11 becomes explicit:

$$
\begin{aligned}
k_{\nu, n} \approx & n \pi \frac{\sqrt{\lambda^{2}-1}}{\lambda-1}+\frac{(\lambda-1) \sqrt{\lambda^{2}-1}}{2 n \pi \lambda} \nu^{2}-\frac{(\lambda-1) \sqrt{\lambda^{2}-1}}{8 n \pi \lambda} \\
+ & \frac{(\lambda-1)^{3} \sqrt{\lambda^{2}-1}}{n^{3} \pi^{3}} Q+\frac{(\lambda-1)^{5} \sqrt{\lambda^{2}-1}}{n^{5} \pi^{5}} P,
\end{aligned}
$$

with

$$
\begin{gathered}
Q \equiv \nu^{4}\left(16 f(\lambda)-\frac{1}{4 \lambda^{2}}\right)+\nu^{2}\left(\frac{1}{8 \lambda^{2}}-104 f(\lambda)\right)-25 f(\lambda)-\frac{1}{64 \lambda^{2}}, \\
P \equiv-\frac{f(\lambda)\left(-25+4 \nu^{2}\right)\left(-1+4 \nu^{2}\right)^{2}}{2 \lambda}+\frac{\left(-1+4 \nu^{2}\right)^{3}}{256 \lambda^{3}} \\
+\frac{\left(-1+\lambda^{5}\right)\left(-1+4 \nu^{2}\right)}{5120(\lambda-1) \lambda^{5}}\left(1073-456 \nu^{2}+16 \nu^{4}\right) .
\end{gathered}
$$

As Eq. (3.1) is asymptotic in $1 / n$, the symbol $\approx$ comes from neglecting all the terms of $\mathcal{O}\left(1 / n^{7}\right)$ and higher. We have defined for convenience

$$
f(\lambda) \equiv\left(\lambda^{3}-1\right) /\left(384 \lambda^{3}(\lambda-1)\right) .
$$

Equation (3.1) contains terms in orders $\mathcal{O}(1 / n), \mathcal{O}\left(1 / n^{3}\right)$, and $\mathcal{O}\left(1 / n^{5}\right)$ that must be taken into account for small $n$ and values of $\lambda \gtrsim 1$. One can solve by numerical methods Eq. (2.2) to obtain the (exact) eigenvalues of the ABAB. This will be done in the next section. Nevertheless, we now want to stress that for fixed values of $\lambda$ and $n>1$, the accuracy of the expansion Eq. (3.1) to the exact eigenvalues depends, not only on $n$, but also on the other quantum number $m$ through $\nu$.

We expand Eq. (3.1) explicitly as a function of both quantum numbers $m$ and $n$. Keeping the terms up to order $\alpha^{2}$ (this is not a crude approximation taking into account that $\alpha$ varies between 0 and $1 / 2$ ) we obtain the corresponding eigenenergies

$$
E_{\nu, n} \equiv E_{m, n}(\alpha) \approx A \alpha^{2}+B \alpha+C+\mathcal{O}\left(\alpha^{3}\right),
$$

with $A, B$, and $C$ polynomials in the quantum number $m$

$$
\begin{gathered}
A \equiv a_{0}+a_{2} m^{2}+a_{4} m^{4}+a_{6} m^{6}+a_{8} m^{8}+a_{10} m^{10} \\
B \equiv b_{1} m+b_{3} m^{3}+b_{5} m^{5}+b_{7} m^{7}+b_{9} m^{9} \\
C \equiv c_{0}+c_{2} m^{2}+c_{4} m^{4}
\end{gathered}
$$


In the Appendix we write down the explicit formulae for the coefficients $a_{i}$ which although rather cumbersome, will be useful in the following. They are analytic functions of the parameter $\lambda$ and the radial quantum number $n$.

The second-order approximation Eq. (3.3) is not the Taylor expansion in $\alpha$ of the eigenenergies. The coefficients in Eqs. (3.4)-(3.6) have been obtained from the asymptotic expansion Eq. (3.1) that, when the radial quantum number $n \leq m$, does not work. This important fact has not been taken into account in Ref. 10. Moreover, even in the limit $n \gg m$ the results of Ref. 10 are, in general, very poor estimates of the eigenenergies. To clarify this point we compare Eq. (3.3) to the equation obtained in Ref. 10 for the eigenenergies, that we have rewritten in the form of Eq. (3.3)

$$
\widetilde{E}_{m, n}(\alpha)=\widetilde{A} \alpha^{2}+\widetilde{B} \alpha+\widetilde{C},
$$

with

$$
\begin{gathered}
\widetilde{A} \equiv \frac{\lambda^{2}-1}{\lambda}, \\
\widetilde{B} \equiv-\left(\frac{\lambda^{2}-1}{\lambda}\right) 2 m, \\
\widetilde{C} \equiv n^{2} \pi^{2}\left(\frac{\lambda+1}{\lambda-1}\right)+\frac{\left(\lambda^{2}-1\right)}{\lambda}\left(m^{2}-1 / 4\right) .
\end{gathered}
$$

Comparing Eqs. (3.4)-(3.6) [and the Eq. (A1) for $a_{i}$ ] to Eq. (3.8) we conclude that, even in the limit $n \gg m$, it is only for $\lambda=1+\epsilon(\epsilon \ll 1)$ that the Eqs. (3.7) and (3.8) give satisfactory values for the eigenenergies of the ABAB. In this case the annulus resembles a thin cylindrical surface of height $(\lambda-1) / \sqrt{\lambda^{2}-1}=R-r$. Let us remark that for a cylindrical surface of area $L \times L_{y}$, the exact eigenenergies are parabolas as a function of $\alpha$. The corresponding coefficients are: $A$ $=(2 \pi / L)^{2}$ (without any dependence on the quantum numbers) for the quadratic term, $B$ $=2 m(2 \pi / L)^{2}$ (depending only on the orbital quantum number $m$ ) for the linear term, and the constant term $C=\left(n \pi / L_{y}\right)^{2}$ which is a function only of the transverse quantum number $n$.

On the contrary, Eqs. (3.4)-(3.6) are valid for larger values of $\lambda$. As an example, Fig. 2 shows a plot of the coefficients $A$ [Eq. (3.4)] and $\widetilde{A}$ as a function of the quantum number $m$ for $\lambda=10$ and $n=30$. In the same figure the empty circles are the exact numerical values for this coefficient (obtained following the prescription that will be describe in the next section). Whereas the coefficient $\widetilde{A}$ only gives the constant value for $m=0$, the behavior of $A$ is quite satisfactory for values of $m \leq 10$. Nevertheless, for $m \leq n$ the coefficients given in Eqs. (3.4)-(3.6) do not reproduce the actual values obtained in the numerical computations. The inclusion of additional terms in these equations does not give a better approximation to the eigenenergies. Under the present condition, Eqs. (3.4)-(3.6) are not perturbative because $|m / n| \geqslant 1$, and any truncation of the expansion is misleading.

The difficulty to obtain a uniform perturbative expansion for the eigenenergies of the $\mathrm{ABAB}$ is related to the very different characteristics of the eigenstates as a function of the quantum numbers. This fact can be understood through semiclassical arguments. Since the problem is integrable, the classical phase space is foliated by tori that are labeled by the values of the actions $I_{i}(l, E), i=1,2$, with $l$ and $E$ the angular momentum and the energy, respectively. ${ }^{12}$ Moreover, this kind of system can be quantized through the E.B.K rule that establishes a correspondence between each eigenstate and a classical torus labeled by $I_{i}=\left(k_{i}+\alpha_{i} / 4\right) \hbar$, where $k_{i}$ is an integer and $\alpha_{i}$ is the Maslov index that depends on the topology of the classical orbits of the system. ${ }^{13}$

Given the values of $l$ and $E$, and according to the ratio $\eta \equiv l \sqrt{\left(\lambda^{2}-1\right) / E}$, we can distinguish two type of classical orbits: Those that do not hit the inner circle $(\eta>1)$, and orbits that hit the inner circle $(\eta<1)$. The special value of $\eta_{c}=1$ corresponds to orbits that are tangent to the inner 


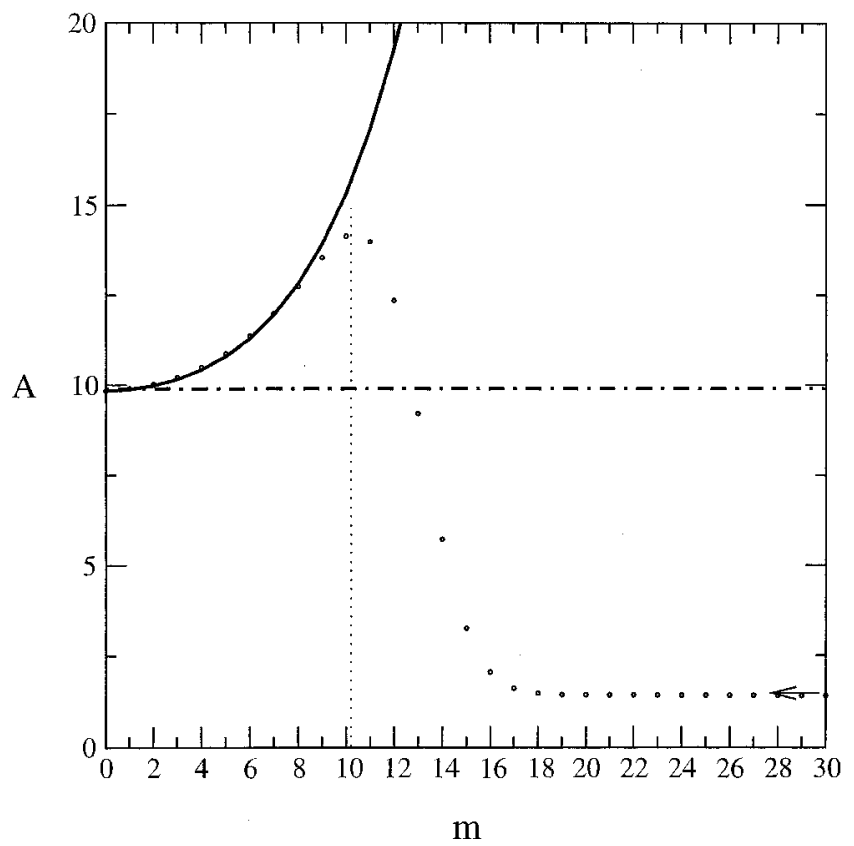

FIG. 2. Coefficient $A$ obtained from Eq. (3.4) (solid lined) and $\widetilde{A}$ obtained from Eq. (3.8) (dotted-dashed line) as a function of the quantum number $m$, for $n=30$ and $\lambda=10$. The empty circles correspond to the numerical values $A_{m n}$ for $n=30$ obtained in Sec. IV. The vertical dotted line is the value $m_{c}$ defined in Eq. (4.3). The left down arrow indicates the analytical estimate $A_{e}$ [Eq. (4.4)] for values of $m>m_{c}$.

circle. The parameter $\eta$ is the ratio between the radius of the caustic of the classical orbit for a disk of radius $R=\lambda / \sqrt{\left(\lambda^{2}-1\right)}$ and the inner radius of the $\mathrm{ABAB}, r=1 / \sqrt{\left(\lambda^{2}-1\right)}$.

According to the preceding remarks, given two values of the quantum numbers $m$ and $n$ the classical motion associated to the quantized torus will correspond either to $\eta>1$ or to $\eta<1$. Therefore, two kind of eigenstates are expected according to the value of $\eta$. Equation (3.3) is valid for quantum states such that $\eta<1$ and becomes a better approximation as $\eta$ decreases.

For $\eta>1$, the classical motion on the ABAB is indistinguishable from that on a disk of radius $R$. Therefore, one would expect that analytical expressions for the eigenvalues corresponding to such a quantum states could be obtained from the Debye's asymptotic expansions for the Bessel functions of the first kind. ${ }^{11}$

However, it is well-known that the Debye's expansions fail to describe states localized on the whispering gallery modes. ${ }^{14}$ In terms on the parameter $\epsilon \equiv \sqrt{E /\left(\lambda^{2}-1\right)} \lambda / l$ the whispering gallery modes correspond to $\epsilon \rightarrow 1$. As $\epsilon$ increases from 1 the Debye's expansion improves. Therefore, the Debye's expansion will be useful to describe states in the ABAB if the conditions $\eta>1$ and $\epsilon$ $>1$ are simultaneously satisfied. As $\eta \cdot \epsilon=\lambda$, the fraction of such states increases with $\lambda$.

Figure 3 shows in the $k$ - $l$ plane the two critical lines labeled $L_{1}$ and $L_{2}$ defined, respectively by the equations $\eta_{c}=1$ and $\epsilon_{c}=1$ for $\lambda=10$. The states lying on the shadowed region in between $L_{1}$ and $L_{2}$ can be approximated by the Debye expansion. As a consequence, while for $\eta<1$ the eigenenergies for the ABAB are given by Eq. (3.1), for $\eta>1$ they could be obtained through the Debye's approximation. In the next section we will explore this approach.

It is important to emphasize that the nature of the failure of the Debye's expansion is quite different from that of the expansion Eq. (3.1). While the former is originated by the pathological behavior of the semiclassical approximation when the classical motion exhibits caustics, ${ }^{14}$ the second one is due to the impossibility of the perturbative expansion Eq. (3.1) to cross the separatrix defined by $\eta=\eta_{c}$. In the next section we will show how this separatrix affects the actual eigenenergies. 


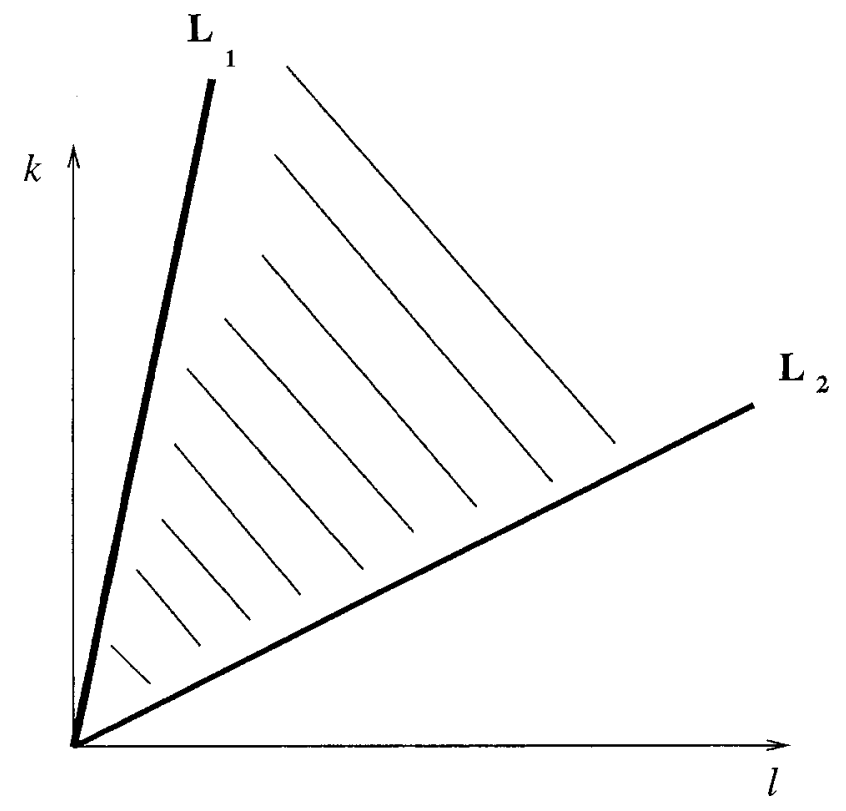

FIG. 3. Critical lines labeled $L_{1}$ and $L_{2}$ defined, respectively, by the equations $\eta_{c}=1$ and $\epsilon_{c}=1$ for $\lambda=10$. The states lying on the shadowed region in between $L_{1}$ and $L_{2}$ can be approximated by the Debye's expansion.

\section{NUMERICAL RESULTS AND ANALYTICAL ESTIMATES}

To obtain each eigenenergy $E_{m, n}$ as a function of the flux $\alpha$, we have numerically solved Eq. (2.2) employing the Newton-Raphson method. For a given value of $\lambda$, we have repeated this procedure for six equally spaced values of $\alpha$ between 0 and 1/2. Then we have fitted the numerical values with a quadratic function that minimizes the sum of the squares of the deviations from the numerical values. Figure 4 shows a region of the energy spectrum as a function of $\alpha$ for $\lambda=10$, obtained by the described procedure. The dotted lines are the quadratic fits, superimposed on the numerical values (circles). In the following we will consider

$$
E_{m, n}=A_{m n}(\lambda) \alpha^{2}+B_{m n}(\lambda) \alpha+C_{m n}(\lambda),
$$

where $A_{m n}, B_{m n}$, and $C_{m n}$ have been obtained from the quadratic fit mentioned above.

To illustrate the statements of the preceding section, we will analize the coefficient $A_{m n}$ as a function of both quantum numbers for two values of the parameter $\lambda$. Figure 5 shows a surface plot of the coefficient $A_{m n}$ as a function of $m$ and $n$ for $\lambda=10$. We can see a pronounced crest separating two plateaux. One of them (labeled as $I$ ) corresponds to values of the quantum numbers $m$ and $n$ such that $\eta<1$. The other plateau (labeled as II) corresponds to $\eta>1$. The values of the coefficient $A_{m n}$ that correspond to eigenstates such that $\eta \approx 1$ are launched on the crest of the surface plot. The crest is a quantum signature of the classical separatrix previously mentioned. For this value of $\lambda$ the fraction of states on the plateau $I I$ is much greater than the fraction of those on the plateau $I$.

Figure 6 shows a surface plot of the coefficient $A_{m n}$ as a function of $m$ and $n$ for $\lambda=2$. The exhibited behavior is qualitatively the same as in Fig. 5. Nevertheless, opposite to the previous case, the fraction of states on the plateau II is smaller than the fraction of those on the plateau $I$.

As we have mentioned in Sec. III, Fig. 2 shows a transverse section of the surface plot Fig. 5 for $n=30$, together with the coefficient $A$ evaluated for $n=30$ [Eq. (3.4)] and the coefficient $\widetilde{A}$ given in Eq. (3.8) that only reproduces the plateau $I(A=9.9$ for the present value of $\lambda=10)$. On the other hand, the coefficient $A$ follows quite satisfactory the numerical values up to the crest of the plot, but it fails to reproduce the behavior of $A_{m n}$ in the complete range of values of $m$. 


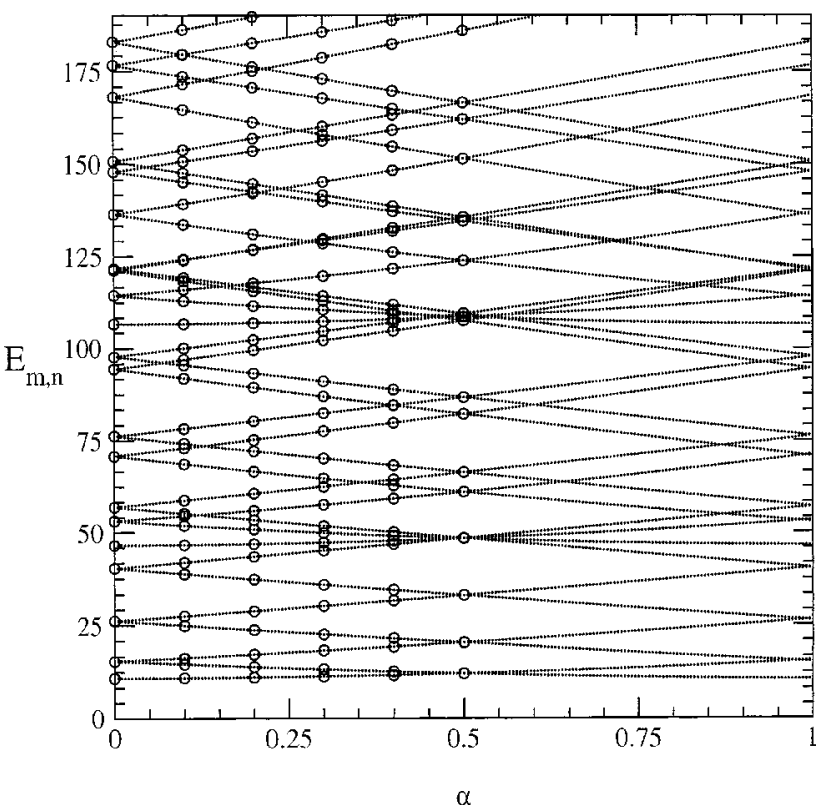

FIG. 4. Single particle energy levels as a function of the adimensional flux $\alpha$, corresponding to the lowest region of the spectrum for the $\mathrm{ABAB}$ with $\lambda=10$. The circles correspond to exact values obtained from the zeros of the cross products of Bessel functions. The small dotted lines are the quadratic fits obtained in Eq. (4.1). See the text for details.

To reproduce the numerical values $A_{m n}$ in the region of the second plateau $I I$ we employ the Debye's approximation ${ }^{11}$ that gives the asymptotic expansion for the zeros of the Bessel functions of the first kind $J_{\nu}(k R)$. If $j_{\nu, n}$ is a zero, $E_{\nu, n}=\left(j_{\nu, n} / R\right)^{2}$ will be the eigenenergy. Expanding $E_{\nu, n}$ as a function of the flux $\alpha(\nu=m-\alpha)$ and keeping the terms up to second order in $\alpha$, we finally obtain

$$
E_{m, n} \sim \frac{1}{R^{2}}\left[\left(\frac{\pi^{2}}{4}-1\right) \alpha^{2}+2 m\left(\frac{\pi^{2}}{4}-1\right) \alpha+\pi^{2}\left(n^{2}+\left(\frac{\pi^{2}}{4}-1\right) m^{2}+\frac{1}{16}\right)\right]
$$

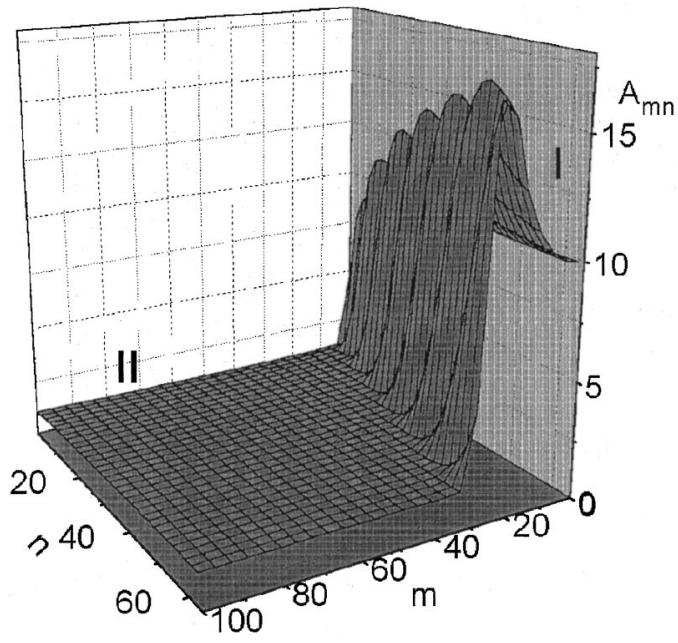

FIG. 5. Surface plot of the coefficient $A_{m n}$ as a function of the quantum numbers $m$ and $n$ for $\lambda=10$. The plateau labeled with I corresponds to values $\eta<1$, whereas the plateau labeled with II to $\eta>1$. 


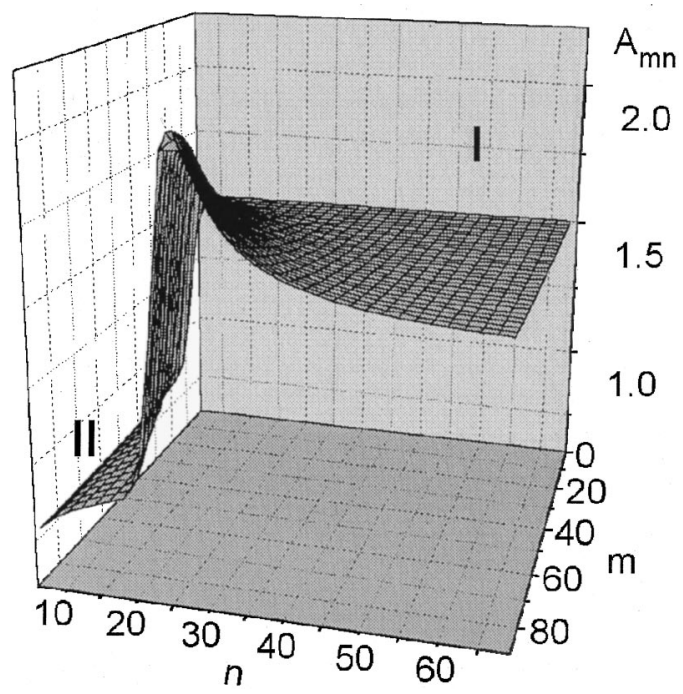

FIG. 6. Surface plot of the coefficient $A_{m n}$ as a function of the quantum numbers $m$ and $n$ for $\lambda=2$. The plateau labeled with I corresponds to values $\eta<1$, whereas the plateau labeled with II to $\eta>1$.

The prefactor in the quadratic term of Eq. (4.2) gives the estimate of $A_{m n}$ in the region of the second plateau $I I(\eta>1)$. For $\lambda=10$ is $R=1.005$ and the value of the coefficient is 1.452 . This value is indicated in the right down part of Fig. 2 by an arrow and it agrees with the numerical values of $A_{m n}$ on the plateau $I I$.

Equation (4.2) can be used to obtain the critical condition $\eta_{c}=1$, in the $n-m$ plane $\left(\eta_{c}=1\right.$ defines the line $L_{1}$ in $k$-l plane). For a given value of the radial quantum number $n, \eta_{c}=1$ determines a critical value for the orbital quantum number $m_{c}$ given by

$$
m_{c} \sqrt{\frac{\lambda^{2}}{\pi^{2}}+1-\frac{\pi^{2}}{4}} \simeq n
$$

where the fact that $m \equiv l$ was used. Figure 7 shows in the $n-m$ plane the critical line defined by the

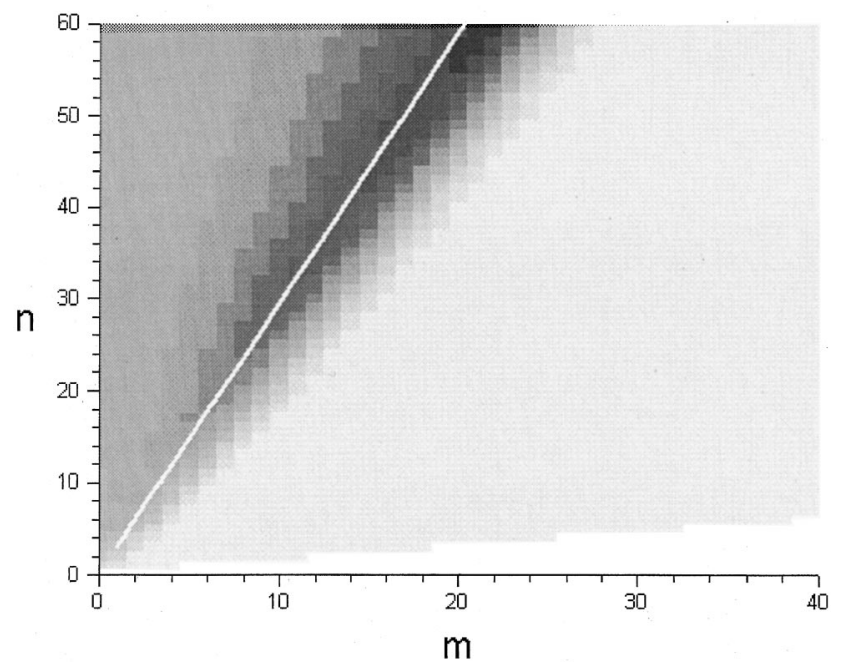

FIG. 7. Gray level density plot in the $m-n$ plane corresponding to the surface plot Fig. 5. The solid white line is the critical line defined in Eq. (4.3) for $\lambda=10$. 
Eq. (4.3) for $\lambda=10$ together with the gray scale density plot of the numerical values of $A_{m n}$ taken from Fig. 5. In the present figure the crest of the surface plot Fig. 5 corresponds to the highest gray level intensity region in where, as expected, the critical line Eq. (4.3) is launched. As a consequence of the preceding analysis, we establish the following functional form to evaluate the coefficients $A_{m n}(\lambda)$

$$
A_{e}=\left\{\begin{array}{l}
\text { A given by Eq. (3.4), for } m \leqslant m_{c} \\
\left(\frac{\pi^{2}}{4}-1\right) \frac{\left(\lambda^{2}-1\right)}{\lambda}, \quad \text { for } m>m_{c}
\end{array},\right.
$$

where $m_{c}$ is given in Eq. (4.3).

\section{CONCLUDING REMARKS}

In the present article we have explored the possibility to obtain an analytical expansion for the eigenenergies of the Aharonov-Bohm annular billiard valid for the whole range of values of the two relevant quantum numbers. We have shown that the well-known expansion for the zeros of the cross products of Bessel functions fail to reproduce the actual behavior of the eigenenergies for some kind of states that are present in all regions of the spectrum (namely for quantum states such that the parameter $\eta>1$ ). Based on semiclassical arguments we have demonstrated that these quantum states are associated with classical orbits that do not hit the inner circle of the annulus. These orbits, when the system is a genuine annulus $(\lambda>1)$, are relevant in all the energy scales. For $\eta>1$ and $\lambda>1$, we have shown that the eigenenergies of the ABAB can be obtained through the Debye's expansions for the zeros of the Bessel functions of first kind, if the condition $\epsilon>1$ is also acomplished. As $\eta \cdot \epsilon=\lambda$, the fraction of states whose eigenenergies can be approximated by the Debye's expansion increases with $\lambda$. We have illustrated our results proposing an analytical estimate $A_{e}$, that reproduces quite satisfactory the exact numerical values $A_{m n}(\lambda)$ at both sides of the critical line defined in $m-n$ plane by the Eq. (4.3), irrespective of the value of the parameter $\epsilon$. That is, although the Debye's expansions fail for $\epsilon \rightarrow 1$, the numerical values $A_{m n}$ are not sensitive to that limit. Therefore, the value predicted by $A_{e}$ for $m>m_{c}$ is valid even in the limit $\epsilon \rightarrow 1$. We should remark that doing analogous calculations to those performed in Sec. IV, it is possible to derive the analytical estimates for the numerical coefficients $B_{m n}$ and $C_{m n}$. This should be equivalent, in view of Eq. (4.1), to find an analytical expansion for the eigenenergies of the $\mathrm{ABAB}$ valid at both sides of the critical line $\eta_{c}=1$.

Last but not least, we would like to remark that the present study is far from being purely academic. The analytical expression of the eigenenergies as a function of the normalized flux $\alpha$ can be employed to determine the actual prefactors in the magnitude of the typical persistent current for a ballistic $\mathrm{ABAB}$ with $N$ carriers, $I_{\mathrm{typ}} \equiv \sqrt{\int_{0}^{1} I^{2} d \alpha}$.

In a recent paper it was found that $I_{\text {typ }} \propto A_{T} / N^{1 / 4}$, being $A_{T} \equiv \Sigma_{m, n} A_{m n}(\lambda){ }^{3}$ Taking into account the analytical expression given in Eq. (4.4) we have obtained, to leading order in $N$

$$
A_{T}=N \frac{\left(\lambda^{2}-1\right)}{\lambda}\left(\frac{\pi^{2}}{4}-1+\frac{\left(8-\pi^{2}\right)(\lambda-1)}{\pi(\lambda+1) \sqrt{4\left(\lambda^{2}+\pi^{2}\right)-\pi^{4}}}\right)+\mathcal{O}\left(N^{1 / 2}\right),
$$

which gives a value of $I_{\text {typ }}$ 1.4-1.5 times larger than the obtained in case of employing Eq. (3.8). This result could help to understand the existent discrepancy between the large experimental values of the persistent current measured in all the experiments performed up to date, and previous theoretical estimates that did not consider the corrections introduced in the present work.

\section{ACKNOWLEDGMENTS}

This work was partially supported by UBACYT (TW35), PICT97 03-00050-01015, and CONICET. 


\section{APPENDIX}

In this Appendix we write down the explicit expressions for the coefficients $a_{m}$ of the different powers of $m$ in the formula Eq. (3.4)

$$
\begin{gathered}
a_{0}=\frac{\lambda^{2}-1}{\lambda}-\frac{(\lambda-1)^{2}\left(\lambda^{2}-1\right)}{8 \lambda^{2} \pi^{2} n^{2}}, \\
a_{2}=\frac{3(\lambda-1)^{2}\left(\lambda^{2}-1\right)}{2 \lambda^{2} \pi^{2} n^{2}}-\frac{69(\lambda-1)^{3}(1+\lambda)}{16 \lambda^{5} \pi^{4} n^{4}}, \\
a_{4}=(\lambda-1)^{3}\left(\frac{3(1+\lambda)}{8 \lambda^{5} \pi^{4} n^{4}} \frac{-1109+4253 \lambda+3812 \lambda^{2}+450 \lambda^{3}+775 \lambda^{4}-935 \lambda^{5}+290 \lambda^{6}}{192 \lambda^{6} \pi^{6} n^{6}}\right), \\
a_{6}=\frac{-7(\lambda-1)^{3}}{720 \lambda^{6} \pi^{6} n^{6}}\left(-41+377 \lambda+188 \lambda^{2}+90 \lambda^{3}+115 \lambda^{4}-155 \lambda^{5}+50 \lambda^{6}\right) \\
+\frac{7(\lambda-1)^{9}}{2880 \lambda^{8} \pi^{8} n^{8}}\left(-423+1897 \lambda+2320 \lambda^{2}\right), \\
a_{8}=\frac{(\lambda-1)^{10}\left(\lambda^{2}-1\right)}{512 \lambda^{10} \pi^{10} n^{10}}\left[-207+1290 \lambda-364 \lambda^{2}+2916 \lambda^{3}-1611 \lambda^{4}\right. \\
+ \\
\left.+2916 \lambda^{5}-3621 \lambda^{6}+1290 \lambda^{7}-207 \lambda^{8}\right] \\
+\frac{(\lambda-1)^{8}\left(\lambda^{2}-1\right)}{64 \lambda^{8} \pi^{8} n^{8}}\left(3-32 \lambda-131 \lambda^{2}-249 \lambda^{3}+131 \lambda^{4}-32 \lambda^{5}+3 \lambda^{6}\right), \\
\quad a_{10}=\frac{(\lambda-1)^{10}\left(\lambda^{2}-1\right)}{9600 \lambda^{10} \pi^{10} n^{10}\left[99-1122 \lambda+6017 \lambda^{2}-17204 \lambda^{3}\right.} \\
\left.+26895 \lambda^{4}-17204 \lambda^{5}+6017 \lambda^{6}+1122 \lambda^{7}+99 \lambda^{8}\right] .
\end{gathered}
$$

${ }^{1}$ L. P. Kouwenhoven et al., Proceedings of the NATO Advanced Study Institute on Mesoscopic Electron Transport, edited by L. P. Kouwenhoven, L. Sohn, and G. Schön (Kluwer Series E, 1997).

${ }^{2}$ K. Richter, D. Ullmo, and R. A. Jalabert, Phys. Rep. 276 (1996).

${ }^{3}$ A. J. Fendrik and M. J. Sánchez, Eur. Phys. J. B 14, 725 (2000).

${ }^{4}$ N. Byers and C. N. Yang, Phys. Rev. Lett. 7, 46 (1961).

${ }^{5}$ M. Büttiker, Y. Imry, and R. Landauer, Phys. Rev. A 96, 365 (1983).

${ }^{6}$ H. F. Cheung, Y. Gefen, E. K. Riedel, and Wei-Heng Shih, Phys. Rev. B 37, 6050 (1988).

${ }^{7}$ H. F. Cheung, Y. Gefen, and E. K. Riedel, IBM J. Res. Dev. 32, 359 (1988).

${ }^{8}$ H. Bouchiat and G. Montambaux, J. Phys. (France) 50, 2695 (1989).

${ }^{9}$ D. Mailly, C. Chapelier, and A. Benoit, Phys. Rev. Lett. 70, 2020 (1993).

${ }^{10}$ R. A. Samandra and W. P. Healy, J. Phys. A 31, 9547 (1998).

${ }^{11}$ M. Abramowitz and I. Stegun, Handbook of Mathematical Functions (Dover, New York, 1972).

${ }^{12}$ A. J. Lichtenberg and M. A. Lieberman, Regular and Chaotic Dynamics (Springer-Verlag, New York, 1992).

${ }^{13}$ M. C. Gutzwiller, Chaos in Classical and Quantum Mechanics (Springer-Verlag, New York, 1990).

${ }^{14}$ P. A. Boasman, Nonlinearity 7, 485 (1994). 